\title{
EQUIDAD EN EL ACCESO A LA EDUCACIÓN SUPERIOR: LOS “CUPOS DE EQUIDAD” EN LA FACULTAD DE CIENCIAS SOCIALES DE LA UNIVERSIDAD DE CHILE
}

\section{Cristóbal Moya ${ }^{1}$}

\section{RESUMEN}

El presente trabajo analiza los "Cupos de Equidad" (CE) como iniciativa que busca formas alternativas de ingreso a la educación superior, en un contexto en el que la selección refleja, en términos socioeconómicos, una alta segregación. La iniciativa es caracterizada a partir de la revisión realizada a algunos documentos que la informan; la cual es cotejada con impresiones provistas por la encargada de su implementación y seguimiento. Finalmente se exponen las fortalezas y debilidades de los CE, de cara a iniciativas internacionales similares. En su desarrollo, el trabajo reflexiona sobre el grado de avance que los CE implican respecto a la equidad en la educación superior, al incluir nuevos estudiantes, mantenga pesar de mantener la exclusión de otros.

Palabras clave: ingreso a la educación superior, discriminación positiva, equidad

\section{EQUITY IN THE ACCESS TO HIGHER EDUCATION: THE "EQUITY PLACES" IN THE SOCIAL SCIENCES CAMPUS OF UNIVERSITY OF CHILE}

\section{ABSTRACT}

This paper analyzes the "Equity Quotas" (CE, for the Spanish acronym) as an initiative that seeks alternative ways of entering higher education in a highly segregated socioeconomic selection context. It analyzes the concept of equity in higher education along with other specific programs and initiatives of alternative access in the last two decades. The initiative is characterized by the revision of some documents that give account of it compared with impressions provided by the person in charge of its implementation and tracing. Finally, the strengths and weaknesses of the $C E$ are exposed, in the light of similar international initiatives. In its development, the work reflects on the level of advance that the CE implies in respect of the equity in higher education, including new students, although it maintains the exclusion of others.

Keyword: access to higher education, positive discrimination, equity

1 Núcleo de Estudios de Artes y Prácticas Culturales. Facultad de Ciencias Sociales, Universidad de Chile. Santiago, Chile. Contacto: cristobalmoya@gmail.com 


\section{EQUIDAD EN EL ACCESO A LA EDUCACIÓN SUPERIOR: LOS "CUPOS DE EQUIDAD" EN LA FACULTAD DE CIENCIAS SOCIALES DE LA UNIVERSIDAD DE CHILE}

\section{Introducción}

Históricamente la educación superior en Chile ha estado enfocada a educar segmentos reducidos de la población, los que además han tendido a provenir principalmente de estratos sociales altos, constituyéndose así en un sistema que bien podemos señalar como elitista. Demandas a todo nivel se han hecho sobre la necesidad de proveer educación terciaria masiva. Por ejemplo, la Declaración de los Derechos Humanos establece en su artículo 26, que "el acceso a los estudios superiores será igual para todos, en función de los méritos respectivos"2. Por otro lado, más recientemente, en Chile se han realizado importantes avances en lo referido a la extensión de la educación primaria y secundaria (García-Huidobro y Bellei, 2003: 58), junto a un explosivo incremento de la oferta de educación superior, lo que ha redundado en que, en los últimos veinte años, el número de matriculados en el nivel se haya multiplicado más de tres veces (Meller y Quiroga, 2010). Se proyecta que la cobertura de educación superior para el grupo etario 18-24 años sea de un 50\% para el 2012.

La institución donde se aloja la iniciativa que se abordará en este artículo tiene características que caben resaltar, en relación con el contexto de instituciones de educación superior. La Universidad de Chile es una institución estatal, pública y de alto prestigio en el país ${ }^{3}$ y que como tal, en el diálogo por el Nuevo Trato para las universidades estatales declara asumir la misión de "proveer opciones de educación superior para todos haciéndose corresponsable en la

2 Declaración de los Derechos Humanos en http://www.un.org/es/documents/udhr/index. shtml, rescatado el 13.12.10.

3 Siendo la única, junto a la PUC, acreditada por siete años (máximo) y en todas las áreas; así como la primera en el país, junto a la PUC, a nivel mundial en rankings internacionales (Shanghai, QS, por ejemplo) 
tarea de superar la desigualdad en la educación en todos sus niveles y crear las condiciones para que esa meta se mantenga en forma permanente" (Pérez, 2009: 4, también en 32). A partir de esta afirmación de rectoría, los mecanismos de selección de estudiantes en las diversas carreras y facultades que componen la Universidad, son revisados poniendo atención al grado de equidad con que estos operan en la práctica.

En este contexto, a comienzos de 2009 surge en el claustro de profesores del Departamento de Psicología de la Facultad de Ciencias Sociales la inquietud de crecer con equidad (Castro, 2010 b: 234). Es así como, al final de ese año, se abrió la convocatoria para postular al "Sistema de ingreso especial de equidad educativa mediante cupos para estudiantes prioritarios", o Cupos de Equidad (CE). Estos cupos consisten en que una carrera asigna un número determinado de cupos para estudiantes de excelencia pertenecientes a los tres quintiles más pobres que hayan cursado su enseñanza media en establecimientos de dependencia municipal ${ }^{4}$.

El presente artículo busca reflexionar respecto de la forma en la cual esta política ha contribuido a la equidad en el acceso a la educación superior desde la perspectiva de lo que la literatura indica como el limitado "ámbito de acción" y efectos de estos tipos de política (McCowan, 2007; Matear, 2006; Gidley, Hampson, Wheeler y Bereded-Samuel, 2010).

En este sentido, resulta fundamental el concepto de equidad, que será discutido más adelante, al constituir el trasfondo discursivo de esta política de acción afirmativa (o discriminación positiva). Precisamente, si consideramos la importancia que varios autores le atribuyen a la educación como factor relevante de la movilidad social (por ejemplo, Torche y Wormald, 2004), preguntarnos por la equidad del acceso a la educación superior constituye un tema social de enorme relevancia, en tanto pareciera haber mucho en juego en ello. Mientras exista la idea de que este nivel de instrucción (que implica haber concurrido a los niveles primarios y secundarios) es

4 Para el año 2011, en la carrera de Psicología, dichos cupos ampararon cerca del 34\% de las matrículas de primer año de la carrera. 
importante por variadas razones, las medidas que se dirijan hacia el desarrollo de mayor equidad en el acceso a ella serán relevantes como objeto de estudio.

Dada la baja existencia de estudios sobre políticas de acción afirmativa en Chile, así como la reciente implementación de esta medida en la FACSO, el presente trabajo tendrá un carácter descriptivo, basado en la revisión de literatura relevante, así como en el análisis de medidas anteriores similares en la educación superior, todo lo cual es cotejado con impresiones provistas por quien ha estado a cargo de la implementación de los CE en la carrera de psicología desde el 2009, en tanto agente clave del proceso.

Así visto, se pretende desarrollar una caracterización de los CE, que permita identificar las dimensiones referidas a la equidad de esta política, así como hacer proyecciones basadas en la comparación con otras medidas similares. Para esto, primero discutiremos distintos desarrollos del concepto de equidad en políticas de educación superior, luego se expondrán políticas similares a las de los CE, así como los antecedentes de esta política en su contexto. Finalmente, se abordarán los CE implementados desde el 2009 en FACSO, con una pretensión reflexiva, revisando con los datos disponibles la forma en que estos cupos se hacen cargo de la equidad en la educación superior, y qué tan limitados son sus efectos dentro del sistema de educación superior.

\section{Concepto de equidad en educación superior}

Si hay algo claro sobre equidad, es que es un concepto complejo y multidimensional. Para McCowan este concepto, en educación superior, tiene dos criterios: primero, deberían existir suficientes puestos en el sistema entero, para que todos los que lo deseen y tengan un mínimo nivel de preparación, puedan participar en la educación superior; segundo, los individuos deben tener una oportunidad justa de obtener un lugar en la institución de su elección (2007: 582). También, destaca la diferencia entre igualdad (equality) y equidad (equity), como lo hará Matear: 
"Mientras igualdad asume un mismo trato para todos, equidad se refiere a justicia y su promoción mediante políticas requiere que las necesidades y condiciones de individuos o grupos sean tomadas en cuenta al planear o diseñar iniciativas. Un enfoque basado en equidad reconoce que mecanismos compensatorios pueden ser requeridos para promover la justicia y para nivelar el campo de juego para grupos desventajados" (2006: 33)

La igualdad, de hecho, atentaría contra la equidad en casos como la realización de procesos de selección iguales para todos, que no toman en cuenta las desventajas que algunos traen por distintas condiciones (socioeconómicas, de raza, género, etc.).

Por otro lado, las políticas de equidad para Gidley et al. representarían un tipo de política pasada, que estarían siendo reemplazadas por las políticas que están bajo el concepto de inclusión social, las que pueden descomponerse, a su vez, en tres niveles: acceso, participación y éxito (2010: 129-131). Dentro del modelo de inclusión social complejo elaborado por los autores, los distintos niveles mencionados corresponden, respectivamente, a tres ideologías: neoliberalismo, justicia social y potencial humano. Para los autores el acceso se identifica con las teorías de libre mercado y de capital humano y social, en tanto las políticas de acceso neoliberales tienen como objetivo principal incrementar la base de habilidades nacionales y mejorar la economía; se trata de números y porcentajes, lo que no refleja necesariamente la participación estudiantil o el logro o éxito de estos, menos dan cuenta de la calidad de la educación a la que se accede (2010: 132). Evidentemente, una política que se quedara solo en este nivel tendría considerables vacíos, y se convertiría en una política con un concepto de equidad dudoso. Sin embargo, todos los autores están de acuerdo en que las políticas enfocadas al acceso más equitativo son un primer paso en la equidad de la educación superior.

5 Traducción propia del original: "While equality assumes sameness of treatment for all, equity refers to fairness or justice and its promotion through policy requires that individuals' or groups' needs and circumstances be taken into account when planning or designing initiatives. An equitybased approach acknowledges that compensatory mechanisms may be required in order to promote fairness and to level the playing field for disadvantaged groups". 
Respecto de las características que el concepto de equidad puede tomar, García-Huidobro (1994) destaca que el argumento de un "trato igual para todos" o de igualdad de oportunidad no es sostenible en el sistema escolar, ya que "La estrategia de tratamiento igualitario resultaría en igualdad si, y solo si, todos los estudiantes fueran iguales"' (213). En tanto se atienda a las diferencias de los estudiantes, especialmente a las creadas por efectos sociales (a nivel agregado), como la condición socioeconómica, sería necesario considerar programas de "acción afirmativa" (afirmative action) o de "discriminación positiva" (positive discrimination) para generar equidad. Ahora bien, esto consistiría en dar un trato desigual con el objetivo de obtener resultados similares en los estudiantes, según García-Huidobro. Es decir, la equidad escolar se mediría en la igualdad de resultados y no en la igualdad de acceso. Más adelante se revisará hacia qué concepto de equidad apuntan los CE y cómo se relacionan con lo expuesto.

\section{Antecedentes}

Los CE están, como hemos mencionado, centrados en el acceso a la educación superior, el que tiene desde el 2003 en Chile un mismo y único sistema de selección (PSU) ${ }^{7}$, que es similar al anterior (PAA) ${ }^{8}$, existente desde 1966. La selección para entrar en los establecimientos de educación superior tradicionales consiste, por lo general, en la ponderación de los resultados de una batería de pruebas PSU, más las notas de enseñanza media (NEM) ${ }^{9}$. Entonces, la primera pregunta es ¿por qué es necesario un método alternativo de acceso a la educación superior? El tema viene dando vueltas, desde principios de los 90, cuando el centro de estudiantes de la Universidad de Santiago de Chile (USACH) denuncia la relación perversa que existía entre PAA y

6 Traducción propia del original: "The strategy of equal treatment would result in equality if, and only if, all pupils were equal".

7 Prueba de Selección Universitaria.

8 Prueba de Aptitud Académica.

9 Hay universidades que piden solo rendir la prueba, sin importar el puntaje, o que hacen sus propias pruebas, y los estudiantes entran mientras puedan costear los aranceles y queden cupos. 
$\mathrm{AFI}^{10}$, en tanto este último instrumento premia la incorporación de los mejores resultados en la PAA, tendiendo estos a ser de establecimientos particulares pagados y subvencionados. Esto desincentivaría abrir la matrícula hacia estudiantes de bajos recursos, los que al tener, en general, bajos puntajes en las pruebas estandarizadas no aportarían AFI a las arcas institucionales.

La USACH, desde 1992 hasta 2008 con la "Iniciativa 4" bonificó en el puntaje de admisión a los estudiantes de establecimientos municipales y particulares subvencionados que estaban dentro de los mejores de sus establecimientos. Respecto a esta iniciativa, estimada como precedente de un programa complementario al ingreso vía prueba estandarizada, sus autores señalan que "en efecto, los resultados del rendimiento académico de los alumnos bonificados son, como era de esperarse, superiores a los de sus homólogos de la promoción que no recibieron la bonificación", además de que "se observa una mejoría general en la calidad académica del estudiantado"11 (Gil y Grez, 2002: 4). El recibir un reconocimiento por el origen escolar es significativo para los beneficiarios, viéndose más motivados que sus pares que entran sin ayuda de esta bonificación. Reconocer la dependencia del establecimiento educacional como fuente relevante de discriminación ${ }^{12}$, dada la alta segregación escolar (Valenzuela, Bellei y de los Ríos, 2010: 14), es preocuparse por las personas de los quintiles más bajos, es decir, las familias de escasos recursos.

La medida que siguió a esta bonificación de la "Iniciativa 4 " en la USACH fue un proyecto que ha tenido gran éxito para los

10 Aporte Fiscal Indirecto, el que consiste en repartir montos de dinero de acuerdo con la distribución de los 27.500 mejores resultados en las pruebas de acceso a la educación superior (y no de acuerdo con las NEM).

11 Otro indicador del éxito de la medida, es el impacto que tuvo en las decisiones de otras instituciones para darles importancia a los estudiantes con mejores notas de sus establecimientos (Gil y Grez, 2002: 4).

12 Las dependencias pueden ser básicamente tres: particular pagado (el establecimiento se autofinancia), particular subvencionado (el establecimiento es administrado por privados, pero recibe subvención del Estado por cada alumno que asiste) y municipalizado (el establecimiento depende de determinado municipio, que recibe la misma cantidad de dinero por alumno que asiste en el caso del particular subvencionado). Además, los dos últimos tipos de establecimientos, con ciertas normativas de por medio, pueden pedir un financiamiento compartido, donde los padres también pagarían una parte, que se suma a la subvención estatal. 
implementadores conocido como "Propedéutico Nueva esperanza, mejor futuro"13. Consiste en tomar a los estudiantes que están entre el $10 \%$ de las notas más altas entre $1^{\circ}$ medio y el primer semestre de $4^{\circ}$ medio de liceos prioritarios de cada curso, y trabajar con ellos los sábados del último semestre de la secundaria (tres a cuatro meses). A los seleccionados, no sólo se les hacen clases de matemáticas y lenguaje (que corresponden a las pruebas obligatorias PSU), sino que también se les hace un taller de gestión personal, a cargo de profesores de Fundación EQUITAS. Las ideas principales detrás de estas dos iniciativas son, según dicen los mismos principios orientadores del sitio oficial del propedéutico, ${ }^{14}$ que "los talentos están igualmente distribuidos entre ricos y pobres", "la educación es una herramienta de fuerte movilidad social, además de proveer mayores oportunidades de desarrollo personal y de convivencia democrática", "las notas de educación media son un mejor predictor de rendimiento académico que las pruebas nacionales de selección como la PSU", "la PSU replica las condiciones de inequidad y exclusión social de la sociedad" y que "hablar de acceso a la educación superior es incompleto, pues la verdadera justicia social se produce al egreso. Se deben aumentar los programas de apoyo que eviten la deserción de los estudiantes, así como respetar las particularidades socioculturales de estos". Buena parte de estas ideas tocan lo referente a la PSU, por lo que es necesario revisar este instrumento de selección.

$\mathrm{Al}$ respecto, un estudio reciente sobre validez diferencial ${ }^{15} \mathrm{y}$ sesgo de la PSU del 2003-2007 ha concluido que "no se encuentra ningún patrón sistemático que evidencie la existencia de validez diferencial" (Comité Técnico Asesor, 2010b: 39). Además, el estudio encuentra leves sesgos que favorecerían a los egresados de establecimientos municipales: "estos resultados se traducen en una

13 Recibió el premio, a fines del 2008, de la Cátedra UNESCO sobre inclusión en educación superior, bautizando el proyecto como "Propedéutico UNESCO". Hasta el 2010 se han sumado otras cinco universidades a este proyecto.

$14 \mathrm{http}: / /$ www.propedeutico.cl//publicacion. aspx?id=2

15 "Los estudios de Validez Diferencial se basan en comparar la magnitud de la relación entre el resultado de la prueba de selección y el rendimiento en la universidad, para diferentes grupos. Cuando el coeficiente de correlación estimado para cada subgrupo es sustancialmente distinto en alguno de ellos, entonces se habla de Validez Diferencial" (Comité Técnico Asesor, 2010: 7). 
ventaja a favor de los establecimientos municipales"16 (2010b: 40). También, las pruebas de matemática aparecen como predictores (del rendimiento del primer año de universidad) tan buenos o mejores que las NEM. De tal manera, los resultados del estudio aparecen como contradictorios con los principios orientadores del propedéutico y de las medidas complementarias y compensatorias que se proponen para la educación superior, pero, como veremos, es posible entender estos resultados como otra arista de las desigualdades en el acceso a la educación superior.

Los datos del estudio pueden ser complementarios, antes que contradictorios, con los programas de discriminación positiva para el acceso. Señalar que los estudiantes de establecimientos municipalizados estén "favorecidos" por la PSU, vale decir que haya una leve sobrepredicción para ellos, significa que en relación con un estudiante con similar puntaje PSU (e igual NEM) de establecimiento particular pagado, el primero tendrá peor desempeño en la universidad. En el fondo, nos dice que hay una diferencia general en la formación que los establecimientos educacionales les dan, según la dependencia de estos (Comité Técnico Asesor, 2010b: 42).

\section{Cupos de Equidad}

El sistema conocido como "Cupo de Equidad", "busca favorecer el ingreso de estudiantes pertenecientes a los tres primeros quintiles de ingreso y provenientes del sector municipal. La comunidad institucional decide implementar esta medida como fórmula correctiva de la tendencia que se apreciaba a la disminución de estudiantes que cumplieran los criterios mencionados anteriormente" (Castro, 2010a: 1). Los datos para la facultad completa y la carrera de psicología se presentan en los siguientes cuadros:

16 Esto dado a que en las comparaciones por dependencia del establecimiento (municipal, particular subvencionado o particular) son las NEM las que constituyen el factor que presenta mayor sesgo predictivo, en este caso, según el estudio, a favor de los establecimientos municipales. 
Gráfico 1. Matriculados en FACSO, por tipo de establecimiento.

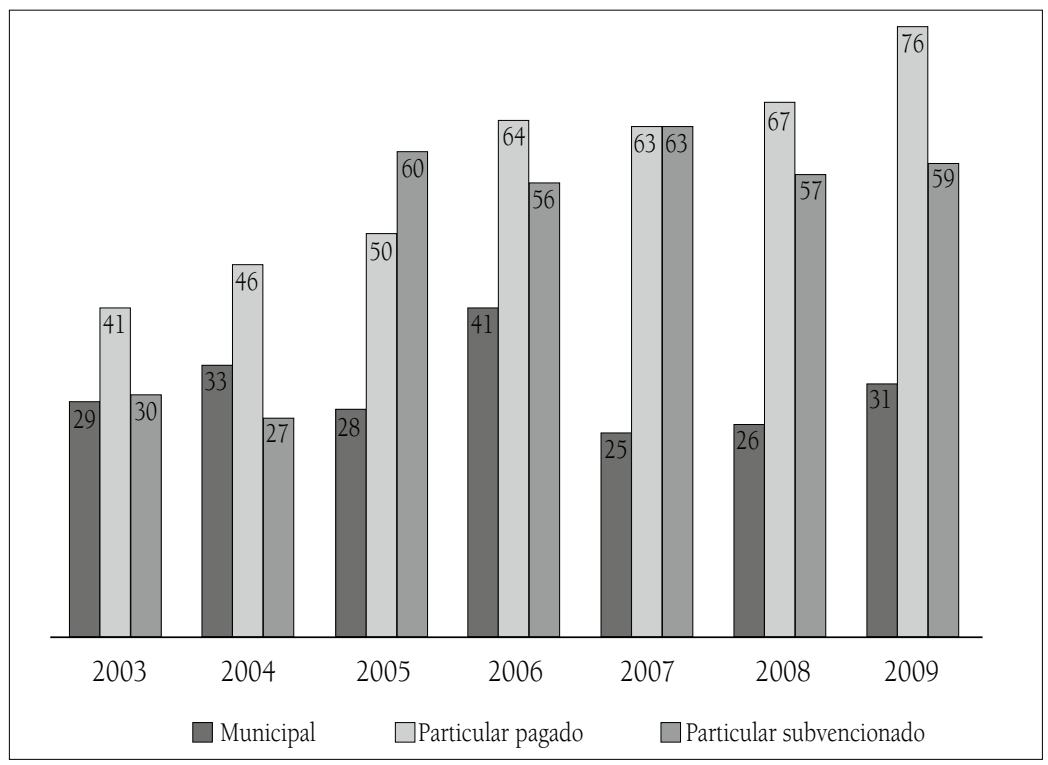

Fuente: Castro, 2010a.

Gráfico 2. Matriculados psicología, por tipo de establecimiento.

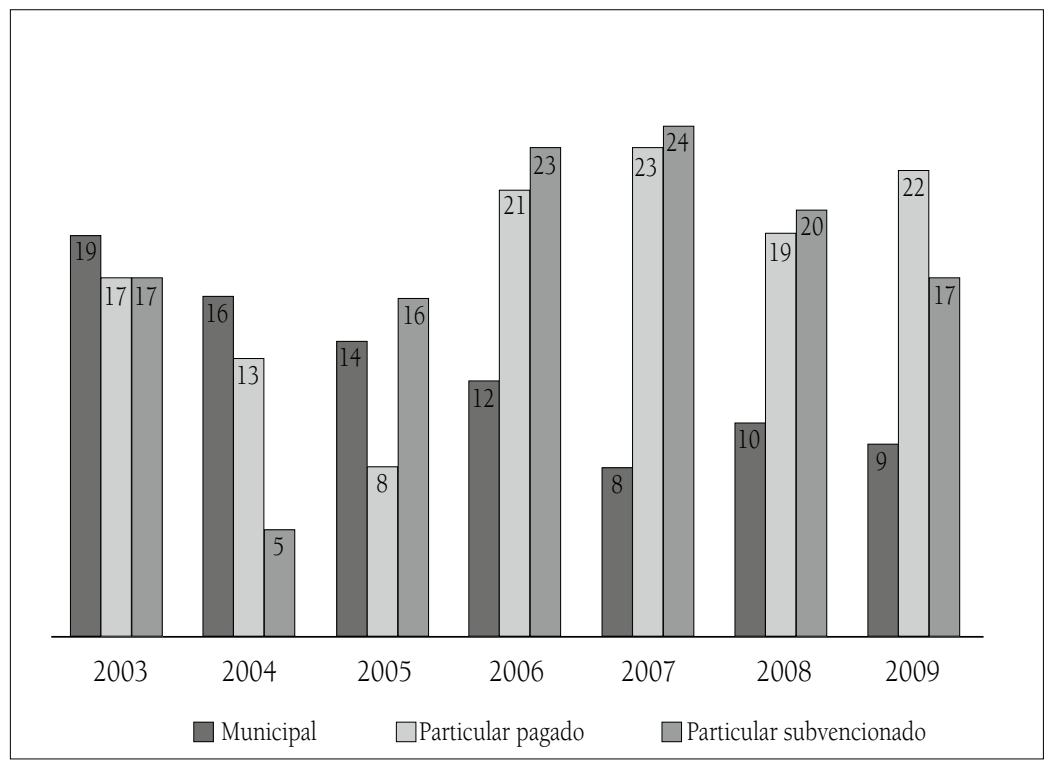

Fuente: Castro, 2010a. 
A finales de 2009 se abre la primera convocatoria solo para la carrera de Psicología de la Universidad de Chile, a la cual llegaron 102 postulaciones, de las cuales 59 cumplían con todos los requisitos. El proceso culminó con los primeros 18 estudiantes que ingresan vía CE a la Facultad de Ciencias Sociales. Estos estudiantes fueron seleccionados a partir de los siguientes criterios:

- Tener un ingreso familiar que los ubicara en los tres primeros quintiles.

- Haber cursado por lo menos los cuatro últimos años de estudio en un establecimiento municipal.

- Haber postulado a los beneficios económicos del Estado (a través del sitio web: becasycréditos.cl).

- Postular el mismo año de egreso de la enseñanza media y en primera preferencia a la carrera de Psicología de la Universidad de Chile.

- Obtener un puntaje, por lo menos, de 600 puntos ponderados en la Prueba de Selección Universitaria (PSU), ${ }^{17}$ que es el mínimo de postulación que exige la Universidad de Chile.

Luego de pasar todos estos filtros, fueron ordenados por puntajes ponderados en la $\mathrm{PSU}^{18}$.

El panorama en el $1^{\text {er }}$ año de Psicología de la promoción 2010 quedó así:

Tabla n. ${ }^{\circ} 1$

\begin{tabular}{|l|c|c|c|c|c|}
\cline { 2 - 6 } \multicolumn{1}{c|}{} & \multicolumn{5}{c|}{$\mathrm{N}^{\circ}$ de matriculados 2010 según tipo de ingreso y establecimiento } \\
\cline { 2 - 6 } \multicolumn{1}{c|}{} & PSU & Cupo BEA & Cupo equidad & Deportista & Total \\
\hline Municipal & 24 & 0 & 0 & 1 & 25 \\
\hline Particular pagado & 18 & 6 & 0 & 0 & 24 \\
\hline Particular subvencionado & 6 & 3 & 18 & 0 & 27 \\
\hline
\end{tabular}

Fuente: Castro, 2010a.

17 La ponderación PSU para la carrera de Psicología corresponde a: un 30\% de las notas de enseñanza media, un $30 \%$ en la prueba de Lenguaje y Comunicación, un $25 \%$ en la prueba de Matemáticas y un 15\% en la prueba específica de Historia y Ciencias Sociales o Ciencias, ya que es obligación por lo menos rendir una de estas dos últimas.

18 Originalmente eran 20 cupos, solo entraron 18 porque un estudiante recibió una oferta de una universidad privada, mientras que el resto postuló a otras carreras del CRUCH en primera opción. 
Desde el punto de vista del equilibrio del origen según dependencia del establecimiento educacional de sus estudiantes, Psicología logró su cometido de "equilibrar el ingreso de sus estudiantes en primer año de acuerdo con el tipo de establecimiento de enseñanza media, rompiendo con esto la tendencia que se apreciaba a lo largo de los años" (Castro, 2010 a: 5), pero ies este equilibrio equitativo? Los colegios particulares pagados constituyen alrededor del 8\% de la matrícula nacional, y tienen un 33\% de representación en esta promoción. Hay que considerar también que, como vemos en el gráfico $n^{\circ} 2$, en los estudiantes que ingresaron el 2009 a la carrera, este porcentaje ascendía a casi un 46\%. La experiencia se considera exitosa, además, porque los estudiantes que ingresaron por esta vía, en general, no solo han aprobado sus asignaturas, sino que su rendimiento es similar al ser comparado con el de estudiantes de los tres primeros quintiles que entraron vía PSU (Castro, 2010 a: 1). Aún así, esto indica que no se produciría el efecto motivacional, que hacía que los estudiantes que ingresaban por cupo o ponderación especial (caso USACH) tuvieran mejores notas que el resto. Esto, quizás, dado el reducido número de estudiantes CE (lo que implica una población insuficiente para hacer un estudio representativo), y la incapacidad de controlar variables que hagan comparables ambos resultados.

La política de CE para el año 2011 permitirá que nuevos estudiantes se incorporen siguiendo los criterios que guían esta medida. Los cupos en psicología se han ampliado, así como se han abierto nuevos cupos en Sociología y Antropología ${ }^{19}$. Hay $26 \mathrm{CE}$ en Psicología, 10 en Sociología y 5 en Antropología. Corresponden aproximadamente al 32\%, $17 \%$ y $11 \%$, respectivamente, de las matrículas de primer año (del total de cupos PSU, BEA ${ }^{20}$ y $\mathrm{CE}^{21}$ ). A

19 La Facultad de Ciencias Sociales tiene una carrera más en pregrado, Educación Parvularia y Básica Inicial. La que no se vio en la necesidad de sumarse a los CE, en tanto está compuesta en buena parte por estudiantes de establecimientos municipalizados.

20 Beca de Excelencia Académica, otorgada por el Estado.

21 Existen otros cupos especiales, como deportivo o de estudiante extranjero, pero no son más de dos por cada uno, y muchas veces no se llenan. 
partir de la reflexión del Departamento de Sociología ${ }^{22}$, se llegó a una nueva ponderación de las postulaciones según la que no castigaría 2 veces por PSU (es decir, solo se exige la barrera de los 600 puntos ponderados), sino que será relevante para la ponderación del ranking de CE el quintil de proveniencia, así como si está o no dentro del 10\% del ranking de su establecimiento. Por último, si hubiera coincidencias en las características, se ordena por el índice de vulnerabilidad del establecimiento de egreso.

Esta medida se plantea entonces como una política de discriminación positiva, asegurando un porcentaje de cupos para los estudiantes de "escasos recursos" que no hayan alcanzado los altos puntajes que piden las carreras de la facultad. Aunque, eso sí, nunca bajo los 600 puntos, que según el Comité Técnico Asesor del CRUCH (2010a: 39), entre el 2006 y el 2010 ha sido alcanzado o superado solo por el 18\% de los que rinden la prueba. Esto introduce un problema fundamental al momento de considerar el programa de los CE: nuevamente aparecería el quintil más alto -ahora aquel con los mejores resultados en la prueba- en la universidad. A lo que debiéramos agregar la fuerte relación que existe entre nivel socioeconómico y resultados en la PSU. Esto es decisivo para acercarnos al concepto de equidad que nos plantean los CE. Bajo el enfoque de equidad descrito por García-Huidobro (1994), esta medida sería confusa, ya que si entendemos la equidad como una igualdad en los resultados, pese a una desigualdad en el acceso, los CE se encuentran en un espacio "intermedio". Como hemos revisado, es una medida que busca ampliar el acceso a estudiantes de grupos socioeconómicos de bajos ingresos, pero con limitantes sustantivas (como los 600 puntos). De tal manera, si consideramos que en el corto tiempo que lleva funcionando el programa, los encargados declaran que en términos de calificaciones no se han constatado mayores diferencias entre estudiantes que ingresen

22 Para el ingreso al año 2011, el comité académico del Departamento de Sociología estableció los siguientes criterios para los CE que ingresen a su carrera: no excluir a los llamados "colegios o liceos emblemáticos", no incluir criterio de etnicidad, ponderar a los quintiles de ingreso y no usar la PSU como criterio de selección final. Esta instancia es solo del Departamento de Sociología, el año anterior esto no estuvo dentro de la discusión, ya que el orden final de los candidatos era según su puntaje en la PSU (una vez que los candidatos habían cumplido el resto de los requisitos). 
vía PSU y vía CE, podríamos tentarnos a adelantar un diagnóstico positivo respecto a la equidad, al encontrarnos frente a similares resultados, no obstante las diferencias en condiciones materiales de vida sustantivas que representarían los distintos y opuestos quintiles a los que pertenecen los estudiantes. Ahora bien, hay dos aspectos para considerar al respecto: los escasos datos disponibles y la barrera del puntaje en la PSU.

Por un lado, es muy pronto para establecer si efectivamente los resultados de los estudiantes no están correlacionados con el grupo socioeconómico del que provienen, ya que ni siquiera hay una generación de estudiantes que haya terminado una carrera habiendo entrado por CE. Por otro lado, y un argumento fundamental al considerar la equidad que estos CE constituyen en la práctica, es que el puntaje mínimo requerido es por sí solo un cuestionamiento para el desarrollo del objetivo deseado. Aceptar este requisito mínimo implica sostener el mismo filtro criticado, que es el de discriminar a estudiantes provenientes de grupos socioeconómicos de bajos ingresos. De tal manera, como hemos dicho, al existir una alta correlación entre puntaje PSU y nivel socioeconómico, donde además los 600 puntos separan al grupo del 18\% con mejor puntaje respecto al universo que rinde la prueba, los CE aparecen rescatando excepciones antes que compensando diferencias creadas por efectos agregados relacionados con los ingresos familiares.

Los CE no proveen ninguna ayuda económica, solo la oportunidad de ingresar, pero es por esto que se hace requisito para postular el haberse inscrito en el sistema estatal de becas y créditos para la educación superior. Lo mismo que los cupos BEA que abre cada carrera, que funcionan independientemente de la PSU, ya que para adjudicarse esta beca hay que estar dentro del 5\% superior del ranking del establecimiento egresado (municipalizado o particular subvencionado) y pertenecer a uno de los cuatro primeros quintiles. Mediante esta política se ha logrado una sinergia entre las medidas estatales para financiar las carreras universitarias de estudiantes de escasos recursos, y el intento por qué estudiantes de excelencia en sus establecimientos secundarios (de bajo nivel de desempeño 
mayormente) puedan acceder a una universidad con las características de la Universidad de Chile ${ }^{23}$.

Es importante tener en cuenta que los implementadores de este programa tienen una preocupación por la proyección del mismo. A partir de una entrevista semiestructurada realizada a la encargada del seguimiento de los $\mathrm{CE}$, que ha estado involucrada desde el principio en el programa, se manifiesta la necesidad del seguimiento y apoyo de los estudiantes que entraron con CE, y del estudiantado en general. Por el momento, esta ayuda y seguimiento consiste, básicamente, en la ayuda de estudiantes mayores ("padrinos" o "madrinas") y profesores, según los que lo necesiten lo pidan voluntariamente. Esto da cuenta de un proceso bastante informal que aún no se hace consistente como estrategia de seguimiento y apoyo. Podemos entender el todavía inconsistente seguimiento tomando en cuenta el proceso desde el que surge la idea hasta su implementación.

Como hemos mencionado, el tiempo de implementación ha sido breve, ya que en menos de un año se llevó a cabo la iniciativa (desde que apareciera la idea en el claustro de Psicología en enero de 2009 hasta la postulación a fines del mismo año). Por lo mismo, las discusiones sobre los diversos aspectos de la iniciativa, como el seguimiento, no han tenido un desarrollo muy avanzado, en aras de una pronta operacionalización. Así, por ejemplo, se cita un recuadro (Castro 2010a) como base del concepto de equidad que es muy confuso al compararlo con la realidad de la implementación de los CE.

23 Que, por efectos de síntesis, pueden ejemplificarse con la posición de la casa de estudios en rankings internacionales, donde ocupa un lugar superior (junto a la Pontificia Universidad Católica de Chile) en relación con otras instituciones universitarias nacionales. Esto le concedería un carácter de prestigio, al menos en el contexto nacional. 
Tabla n. ${ }^{\circ} 2$

\begin{tabular}{|c|c|c|c|c|c|}
\hline \multirow[t]{2}{*}{ Concepto } & \multicolumn{5}{|c|}{$\begin{array}{l}\text { Modelo de equidad en la educación superior } \\
\text { Etapas del proceso educativo }\end{array}$} \\
\hline & Recursos & Acceso & Permanencia & Logros & Resultados \\
\hline Equidad & $\begin{array}{l}\text { Garantizar } \\
\text { que todas } \\
\text { las personas } \\
\text { que tienen } \\
\text { las mismas } \\
\text { necesidades, } \\
\text { capacidades } \\
\text { y logros } \\
\text { obtengan la } \\
\text { misma cantidad } \\
\text { de recursos } \\
\text { financieros, } \\
\text { sociales y } \\
\text { culturales. }\end{array}$ & \begin{tabular}{|l} 
Permitir que \\
todos los \\
individuos que \\
tengan similares \\
necesidades, \\
capacidades y \\
logros tengan \\
acceso a una \\
educación de \\
calidad.
\end{tabular} & $\begin{array}{l}\text { Resguardar que } \\
\text { los estudiantes } \\
\text { con iguales } \\
\text { necesidades, } \\
\text { capacidades } \\
\text { y logros } \\
\text { permanezcan } \\
\text { en el sistema. }\end{array}$ & $\begin{array}{l}\text { Salvaguardar } \\
\text { que los } \\
\text { estudiantes } \\
\text { con iguales } \\
\text { necesidades, } \\
\text { capacidades } \\
\text { y éxitos } \\
\text { académicos } \\
\text { anteriores } \\
\text { obtengan logros } \\
\text { similares en } \\
\text { términos de } \\
\text { calificaciones. }\end{array}$ & $\begin{array}{l}\text { Asegurar } \\
\text { que aquellos } \\
\text { individuos } \\
\text { con iguales } \\
\text { necesidades, } \\
\text { capacidades y } \\
\text { logros obtengan } \\
\text { similares } \\
\text { empleos, nivel } \\
\text { de ingresos y } \\
\text { poder político. }\end{array}$ \\
\hline
\end{tabular}

Fuente: Latorre, C., González, L., Espinoza, O. 2009.

Respecto de "resultados" no existe ningún lineamiento que apunte directamente a actuar o atender a este aspecto dentro de la actual estructura de la iniciativa CE, así como la operacionalización de conceptos como capacidades y éxitos no está debidamente fundamentada. Podemos suponer, en este caso, que la motivación por una rápida "puesta en marcha" de la iniciativa dejó en un plano secundario una discusión más profunda sobre el proyecto.

Sobre la implementación de los CE, proceso que siguió íntegramente la entrevistada, señala: “...Si se instaló, si se logró instalar acá, fue porque la pelea política fue importante, y como había un claustro de profesores que estaba dispuesto a darla, se pudo instalar...", con lo que destaca la participación de estudiantes y profesores a favor de la implementación. Agrega que: “...Yo soy un peón más en esta institución... yo no soy una académica importante, yo no tengo un cargo importante, yo soy un engranaje más", con lo cual parece destacar que la implementación de esta política dependería en buena parte de diálogos políticos desarrollados al interior de las restricciones institucionales particulares de cada unidad educativa. 
Por lo mismo, los CE son todavía una medida local, que aún no resuena a nivel institucional en la Universidad de Chile, lo que está relacionado con su alto nivel de burocratización, la que opera como constreñimiento en este caso. Sobre este aspecto, es relevante resaltar que los CE existen en virtud del artículo no 6 letra d) del Reglamento General de Estudiantes de Pregrado, que permite ingresos por sistemas especiales de selección mientras que las personas estén "amparadas por convenios suscritos por la Universidad de Chile con otros organismos públicos o privados", lo que significó firmar un convenio con la Asociación Chilena de Municipalidades (representando a todos los municipios del país). De alguna forma, fue este "resquicio legal" el que permitió la rápida implementación de los CE, superando el importante grado de burocratización de la Universidad, que marca límites considerables a cualquier discusión y/o consenso político al interior de esta ${ }^{24}$.

\section{Conclusiones y proyecciones}

La mayoría de las medidas mencionadas parecen plantearse contra el método de selección de la educación superior (PSU), dejando un poco de lado la crítica a los resultados de colegios municipalizados. Más allá de la discusión de dónde está el problema, si en la PSU o en la educación que se recibe en los establecimientos $-y$, probablemente, la respuesta es una mezcla de las dos-, el concepto de equidad que se construye con esta política de CE apunta a un tipo de dessegregación (poniendo porcentajes equivalentes de estudiantes de las tres dependencias en el $1^{\text {er }}$ año de la carrera), pero que es parcial, limitando incluso lo que aparece como un primer nivel en políticas de inclusión social, como es el acceso (Gidley et al., 2010: 129-131). Evidentemente, es una política muy joven que tiene puntos críticos bien diagnosticados por sus implementadores, como la necesidad del seguimiento y apoyo de los estudiantes que entraron con CE y del estudiantado en general, como se mencionó con anterioridad.

24 Esto podría asociarse a lo que Blossfeld y Shavit (2000) ven en un nivel macro, pero en lo micro como esta política. Según los autores, existe una asociación importante entre las políticas y reformas con los cambios a nivel social, aunque claramente no tenemos datos suficientes para avanzar más en esta línea. 
La rapidez de la implementación, así como el diálogo más político que intelectual/académico, puede explicar el modelo de equidad más concreto con que se trabaja en este sistema de $\mathrm{CE}^{25}$. No parece existir una solución satisfactoria a un concepto claro y preciso de equidad, pero la evidencia empírica da luces sobre la dimensión operacional de este concepto: se busca pluralidad social (partiendo por las diferencias de nivel socioeconómico), en tanto los datos disponibles dan un diagnóstico sobre una mayoría excluida del sistema de educación superior. Curiosamente, cuando se habla sobre estas medidas de discriminación positiva se piensa generalmente en "minorías". En Australia ${ }^{26}$ y Brasil hay políticas en esta línea, las políticas de cuotas de este último país tienen varias similitudes con el caso CE chileno, a excepción de que allá existen universidades públicas y gratuitas. También, existe oposición de varios sectores del espectro político, así como hay un consenso en los que están a favor sobre el carácter "provisional" de esta medida (McCowan, 2007: 591). La equidad que los CE proponen es de un nivel inicial, que busca que no solo quepan más estudiantes en el aula, sino que sean también otros los estudiantes que cabrán en la facultad. Se busca abrir la posibilidad hacia otros estudiantes, en el entendido de que sean de orígenes socioeconómicos diversos y se destaquen en sus establecimientos educacionales de origen, pero siempre con un mínimo -bastante alto- de puntaje en la PSU, lo que, en este caso, termina generando una política de excepciones antes que una medida compensatoria. Así es como la facultad se plantea "crecer, pero crecer con equidad", dentro de las restricciones institucionales de una universidad altamente burocrática y en un contexto donde el resguardo por la calidad (representado en el alto del corte de los puntajes PSU) entra en contradicción con el objetivo de ampliar el acceso. Esto dado que el puntaje en la prueba de selección sigue siendo requisito, pese a como hemos mencionado, la constatación de una alta correlación entre puntaje PSU y nivel socioeconómico.

25 Expuesto en el recuadro anterior.

26 "Access to success" es un proyecto con cinco años que busca mejorar el acceso y la participación exitosa de jóvenes de grupos de bajos ingresos y minorías, en conjunto con escuelas en el oeste de la región de Melbourne. 
Entonces, sobre el alcance de los CE pareciera que, siendo una iniciativa de esta escala, está fuertemente limitada por los constreñimientos institucionales y sociales, pero que dentro de estos ha logrado un avance en la Universidad y en la sociedad en general, en parte como un gesto político y en parte como una iniciativa, atreviéndose a incluir más estudiantes en sus planteles con miras a la equidad. Esto es, en buena parte, mostrar una señal de que la universidad no simplemente recibe matrículas (y aranceles), sino que toma la responsabilidad de incluir y retener a sus estudiantes (Meller y Quiroga, 2010: 46).

Se debe tener especial cuidado en cómo se hará para asegurar la mayor retención posible de estos estudiantes, sobre todo si tomamos en cuenta el dato del estudio realizado por la UNESCO ${ }^{27}$ el año 2006, según el cual los estudiantes de los dos quintiles de menores recursos en los primeros cuatro años de carreras tienen tasas de deserción que superan en más de dos veces las tasas de los estudiantes de los dos quintiles de mayores ingresos. Sumado a la rapidez con que se ha implementado la iniciativa, es de mayor importancia que se evalúen las actuales formas de seguimiento y apoyo. Por otro lado, es fundamental revisar el requisito de puntaje, ya que marca una diferencia fundamental en el tipo de equidad a la que se aspira en la práctica, al transformar la iniciativa en una selección de excepciones, antes que en una compensación a las condiciones materiales desfavorables en que se encuentran los estudiantes, representada por su nivel socioeconómico.

Por último, sobre la importancia que se le atribuye a la educación superior, está por verse el efecto a mediano y largo plazo de políticas de este tipo, en tanto puedan producir un cambio en las formas de movilidad social, o solo lleven a una sobreeducación, generando una movilidad relativa donde un joven que estudie cinco o seis años más que su padre, termine en la misma posición que este o peor. Una observación de este tipo desborda los alcances del presente trabajo, se presenta no solo como un horizonte crítico que debe revisarse más allá de las fronteras nacionales, sino que en el

27 Citado en Meller y Quiroga, 2010. 
conjunto de las recientes iniciativas por hacer de la educación superior un sistema más equitativo, con mayor inclusión social.

\section{Referencias bibliográficas}

Blossfeld, H. \&Y. Shavit. (2000) "Persisting Barriers. Changes in Educational Opportunities in Thirteen Countries". En The Structure of Schooling. R. Arum \& I. Beattie (comp.), McGraw-Hill.

Castro, M.P. (2010a) Cupo de equidad en la carrera de psicología de la Universidad de Chile: Una experiencia que invita a cuestionar la PSU como criterio fundamental de selección y predicción del éxito académico en la educación superior. Sin publicar.

Castro, M.P. (2010b) "Ingreso especial de equidad educativa: Mayor diversidad, una oportunidad para la carrera de psicología de la Facultad de Ciencias Sociales de la Universidad de Chile". En Diagnóstico y Diseño de Intervenciones en Equidad Universitaria. Santiago de Chile: CINDA.

Comité Técnico Asesor CRUCH. (2010a) "Resultados de la aplicación de pruebas de selección universitaria. Admisiones 2006-2010". Documentos Técnicos. Santiago de Chile: CRUCH.

Comité Técnico Asesor CRUCH. (2010b) "Validez diferencial y sesgo de predictividad de las pruebas de admisión a las universidades chilenas". Documentos Técnicos. Santiago de Chile. CRUCH.

García-Huidobro, J.E. (1994) "Positive Discrimination in Education: Its Justification and a Chilean Example", International Review of Education Vol. 40, n 3/5 (enero 1). pp. 209-221.

García-Huidobro, J. E. y Bellei, C. (2003) "Desigualdad educativa en Chile". Documento de Trabajo, Universidad Alberto Hurtado.

Gidley, J.; Hampson, G.; Wheeler, L. y Bereded-Samuel, E. (2010) "From Access to Success: An Integrated Approach to Quality Higher Education Informed by Social Inclusion Theory and Practice". Higher Education Policy 23, 123-147. doi: 10.1057/hep.2009.24.

Gil, F. y Grez, N. (2002) "Igualdad de oportunidades en el ingreso a las universidades". En Conocer y situar al estudiante reto a la calidad no 6 . Santiago de Chile: Consejo Nacional de Educación.

Gil, F, y Bachs, J. (eds.). (2009) Propedéutico USACH/UNESCO. Una experiencia exitosa por una educación superior más inclusiva. Santiago de Chile.

Latorre, C.L.; Espinoza, O. y González, L.E. (2009) "Un modelo de equidad 
para la educación superior: Análisis de su aplicación al caso chileno". Revista de la Educación Superior (ANUIES), nº 150 (Abril-Junio), Ciudad de México, México.

Madera, A. (2006) "Barriers to Equitable Access: Higher Education Policy and Practice in Chile Since 1990". Higher Education Policy, 19. p. 31-49.

McCowan, T. (2007) "Expansion Without Equity: An Analysis of Current Policy on Access to Higher Education in Brazil". Higher Education: The International Journal of Higher Education and Educational Planning, v. 53, $\mathrm{n}^{\circ}$ 5, p 579-598, mayo.

Meller, P. y Quiroga, V. (2010) "Evolución de la educación universitaria". En Meller, Patricio Carreras universitarias: estudios sobre economía de la educación superior. Santiago de Chile: Uqbar.

Pérez, V. (2009) "Nuevo trato con el Estado. Hacia una política para las universidades estatales". Documento de trabajo.

Torche, F. y Wormald, G. (2004) "Estratificación y movilidad social en Chile: entre la adscripción y el logro". Serie Políticas Sociales No 98, CEPAL.

Valenzuela, J.P.; Bellei, C. y de los Ríos, D. (2010) "Segregación escolar en Chile". En Martinic, S. y Elacqua, G. (eds.) Cambios en la gobernanza del sistema educativo chileno. Santiago de Chile: UNESCO y Pontificia Universidad Católica de Chile.

Recibido: 27/04/2011

Aceptado: 14/11/2011 